$$
\text { Pontifícia Universidade C Católica }
$$

Alexandre Vargas Grillo

\title{
Produção e Caracterização de Nano Partículas do Sistema Ti-N-O via Reação na Fase Vapor
}

\section{Dissertação de Mestrado}

Dissertação apresentada como requisito parcial para obtenção do título de Mestre pelo Programa de PósGraduação em Engenharia Metalúrgica do Departamento de Ciência dos Materiais e Metalurgia da PUC-Rio.

Orientador: Prof. Francisco José Moura Co-Orientador: Prof. Ivan Guillermo Solórzano Naranjo

Rio de Janeiro

Setembro de 2008 


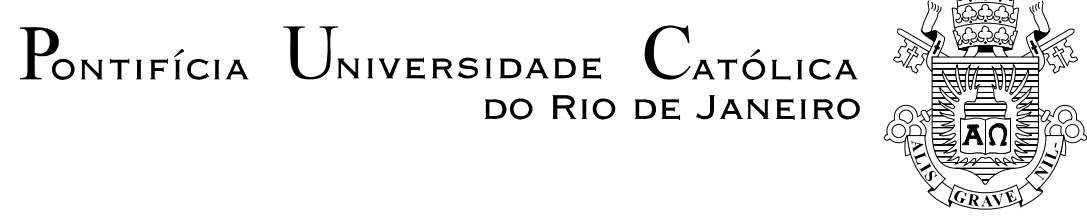

Alexandre Vargas Grillo

\section{Produção e Caracterização de Nano Partículas do Sistema \\ Ti-N-O via Reação na Fase Vapor}

Dissertação apresentada como requisito parcial para obtenção do grau de Mestre em Engenharia Metalúrgica e de Materiais pelo Programa de Pós-Graduação em Engenharia Metalúrgica do Departamento de Ciência dos Materiais e Metalurgia do Centro Técnico Científico da PUCRio. Aprovada pela Comissão Examinadora abaixo assinada.

Dr. Francisco José Moura

Orientador e Presidente

Pontifícia Universidade Católica do Rio de Janeiro - PUC-Rio

Dr. Ivan Guillermo Solórzano Naranjo

Co-Orientador

Pontifícia Universidade Católica do Rio de Janeiro - PUC-Rio

Dr. Roberto José de Carvalho

Pontifícia Universidade Católica do Rio de Janeiro - PUC-Rio

Dr. Bruno Cavalcante Di Lello

Universidade Estácio de Sá - UNESA

Prof. José Eugenio Leal

Coordenador Setorial de Pós-Graduação do Centro Técnico Científico

PUC-Rio

Rio de Janeiro, 12 de setembro de 2008 
Todos os direitos reservados. É proibida a reprodução total ou parcial do trabalho sem autorização da universidade, do autor e do orientador.

\section{Alexandre Vargas Grillo}

Graduou-se em Engenharia Química na Pontifícia Universidade Católica do Rio de Janeiro (PUC-Rio) em 2005.

Ficha Catalográfica

Grillo, Alexandre Vargas

Produção e caracterização de nano partículas do sistema Ti-N-O via reação na fase vapor / Alexandre Vargas Grillo ; orientador: Francisco José Moura ; co-orientador: Ivan Guillermo Solórzano Naranjo. - 2008.

89 f. ; $30 \mathrm{~cm}$

Dissertação (Mestrado em Ciência dos Materiais e Metalurgia) - Pontifícia Universidade Católica do Rio de Janeiro, Rio de Janeiro, 2008.

Inclui bibliografia

1. Ciência dos materiais e metalurgia - Teses. 2 . Nitreto de titânio. 3. Dióxido de titânio. 4. Nanopartículas. 5. Síntese. 6. Caracterização. I. Moura, José Francisco. II. Solórzano, Guillermo. III. Pontifícia Universidade Católica do Rio de Janeiro. Departamento de Ciência dos Materiais e Metalurgia. IV. Título.

CDD: 669 
Dedico esta dissertação a minha querida mãe Estela Vargas Grillo, meu pai Vincenzo Grillo, meu padrasto Jorge Luiz Zaupa, minha irmã Denise Vargas Grillo e meu amigo, mestre e orientador Francisco José Moura, pelo grande incentivo e amor. 


\section{Agradecimentos}

Ao meu orientador e amigo, Professor Francisco José Moura pelo estímulo, paciência e parceria em todos os momentos da pesquisa, por sua amizade e ilimitada vontade de me auxiliar em todos os momentos, da graduação à pósgraduação.

Ao professor e co-orientador, Guillermo Solórzano pela ajuda durante as análises por microscopia eletrônica de transmissão e de varredura.

Ao Professor e amigo Bruno Cavalcante Di Lello pelo estímulo, amizade e parceria em todos os momentos da pesquisa.

A minha amiga e Doutora Orfelinda Avalo Cortez e ao mestre e amigo, Martin Emilio Mendoza Oliveros, pela enorme ajuda e companheirismo durante o estudo.

Aos meus amigos da PUC-Rio, Carlos Augusto Ribeiro Queiroz, Rosangela Magnani Diogo, Cosme Pereira Leal, Wilson de Almeida Guerra, Javier Antahuara Lazo e Ysrael Marrero.

Aos meus amigos de infância, Aurélio da Silva Regueiro, Carlos Adriano, Cristiano Rebello Menendes, Kennedy Fernandes e Carlos Alberto Lopes Quintãs pela grande amizade e incentivo.

Como Deus me concedeu uma gama enorme de amigos, agradeço também aos meus grandes amigos: André Luiz dos Santos Pazza, Bernardo D'aragona, Bruno Cananéa Lopes, Fabiano Gabry de Castro, Gustavo Leite Machado, Gustavo Pilotto Domingues Sá, Leonardo Liberato, Marcelo Couto Ferreira, Máximo de Carvalho Gonçalves, Nuno Virgílio Neto, Pedro Gonçalves Coppos, Pedro Gualda, e Wander Ricardo Ferreira Lopes.

Obrigado a todos vocês.

Ao CNPq - Conselho Nacional de Pesquisa, pelo apoio financeiro na realização deste trabalho. 


\section{Resumo}

Alexandre Vargas Grillo; Moura, José Francisco (Orientador). Produção e caracterização de nano partículas do sistema $\mathrm{Ti}-\mathrm{N}-\mathrm{O}$ via reação na fase vapor, Rio de Janeiro, 2008. 89p. Dissertação de Mestrado Departamento de Ciência dos Materiais e Metalurgia, Pontifícia Universidade Católica do Rio de Janeiro.

Os nitretos, especialmente os de metais de transição, têm recebido nos últimos anos, grande atenção, devido às suas propriedades físicas e químicas serem únicas. Dentre estes nitretos, temos o nitreto de titânio (TiN) que apresenta muitas propriedades interessantes, que fazem com que tenha grandes potencialidades para aplicações à nível industrial. A importância do nitreto de titânio (TiN) se deve às suas propriedades de alta condutividade térmica, dureza, resistência ao desgaste, alta condutividade elétrica e não é tóxico. Assim, a sua principal área de uso, está relacionada como um material que apresenta aplicações nos campos químicos e mecânicos. $\mathrm{Na}$ indústria aeroespacial, é utilizado em equipamentos, como turbinas e motores a jato, e, no ramo da medicina, sob a forma de revestimento em próteses ortopédicas, válvulas cardíacas e próteses dentárias. O objetivo desta dissertação é estudar a produção e a caracterização de nano partículas de nitreto de titânio (TiN), a partir de uma reação na fase gasosa entre o tetracloreto de titânio $\left(\mathrm{TiCl}_{4}\right)$ e a amônia $\left(\mathrm{NH}_{3}\right)$ em um reator tubular, e a influência dos parâmetros reacionais (temperatura e tempo espacial), sobre o tamanho médio das partículas. Os resultados experimentais mostraram que as variações destes parâmetros produziram pós de TiN com diferentes tamanhos de partículas. Além da produção de pó de nitreto de titânio (TiN), constatou-se a presença de cloreto de amônio $\left(\mathrm{NH}_{4} \mathrm{Cl}\right)$. Durante o manuseio do pó de TiN produzido, houve a formação de $\mathrm{TiO}_{2}$ (anatásio) através da reação do primeiro com o ar atmosférico.

\section{Palavras-chave}

Nitreto de Titânio; Dióxido de titânio; Nanopartículas; Síntese; Caracterização. 


\section{Abstract}

Alexandre Vargas Grillo. Production and characterization of TiN nano particles from vapor phase, Rio de Janeiro, 2008. 89p. MSc Dissertation - Departamento de Ciência dos Materiais e Metalurgia, Pontifícia Universidade Católica do Rio de Janeiro.

Nitrides, especially those of transition metals, have received increasing attention in recent years because of their unique chemical and physical properties. Among them, TiN presents many interesting properties, which make it interesting for some potential industrial applications. The importance of nitride titanium (TiN) is due to its properties of high thermal conductivity, chemical inertial, hardness, wear resistance, high electrical conductivity and nontoxicity. The objective of this master thesis is the production of TiN powder from gas phase reaction between titanium tetrachloride $\left(\mathrm{TiCl}_{4}\right)$ and ammonia $\left(\mathrm{NH}_{3}\right)$ in a tubular reactor and the study of the effects of the reaction parameters, temperature and space time, on particle size. The experimental results showed that the variation of these parameters produced nanoparticles of TiN with different sizes, heterogeneous. Besides the production of titanium nitride powder, there was also the presence of ammonium chloride $\left(\mathrm{NH}_{4} \mathrm{Cl}\right)$, co-product of the nitridation reaction. During the handling of the TiN powder occurred the formation of $\mathrm{TiO}_{2}$ due to its reaction with atmospheric air.

\section{Keywords}

Titanium nitride; titanium dioxide; synthesis; nanoparticles; characterization. 


\section{Sumário}

1 INTRODUÇÃO

2 OBJETIVO DO TRABALHO 15

3 REVISÃO DA LITERATURA 16

$\begin{array}{ll}\text { 3.1. Materiais } & 16\end{array}$

3.2. Nanopartículas 17

3.3. Titânio 18

3.3.1. Propriedades 19

3.3.2. Ocorrência 20

3.3.3. Aplicações 20

3.3.4. Reservas 20

3.3.5. Mercado 22

3.4. Propriedades do Nitreto de Titânio (TiN) 22

3.4.1. Aplicações 23

3.5. Propriedades do Dióxido de Titânio $\quad 24$

3.6. Métodos de Síntese de Nitreto de Titânio 25

3.6.1. Método CVD (Chemical Vapour Deposition) 26

3.6.2. Método PVD (Physical Vapour Deposition) 27

3.6.3. Aplicações PVD e CVD 27

3.6.4. Método de Síntese por Combustão Auto-Sustentada a Alta

3.7. Outros Métodos 29

3.8. Síntese de pós-cerâmicos a partir da fase vapor 31

4 CONSIDERAÇÕES TERMODINÂMICAS 36

4.1. Estudo termodinâmico da Vaporização do Tetracloreto de Titânio $\left(\mathrm{TiCl}_{4}\right) \quad 36$

4.2. Estudo termodinâmico para a determinação da temperatura do forno 37

4.3. Estudo termodinâmico de Formação do Nitreto de Titânio 38

4.4. Estudo termodinâmico de Formação do Dióxido de Titânio $\left(\mathrm{TiO}_{2}\right) \quad 40$

4.5. Estudo da Decomposição da Amônia $\left(\mathrm{NH}_{3}\right)$

4.6. Formação do Cloreto de Amônio 43

4.7. Formação do Pó Cerâmico 44

4.7.1 Nucleação de Partículas 44

4.7.2. Crescimento das Partículas $\quad 45$

4.7.3. Aspectos cinéticos da Coagulação Browniana 47

5 MATERIAIS E MÉTODOS $\quad 49$

5.1. Equipamento Experimental e Materiais 49

5.2. Materiais 53

5.2.1. Tetracloreto de titânio - $\mathrm{TiCl}_{4}$

5.3. Método Experimental $\quad 54$

5.4. Parâmetros Experimentais $\quad 55$

5.5. Caracterização do Material Sintetizado $\quad 56$

5.5.1. Difração de Raios-X $\quad 56$

5.5.2. Microscopia Eletrônica de Transmissão (MET) 57

5.5.3. Microscopia Eletrônica de Varredura (MEV) 58 
6 RESULTADOS E DISCUSSÃO 60

6.1. Análise do pó sintetizado de TiN por Difração de Raios-X 60

6.1.1. Análise do pó sintetizado de TiN - Sem Tratamento Térmico 60

6.1.2. Análise do pó sintetizado de TiN - Com Tratamento Térmico 62

6.2. Efeitos dos Parâmetros Reacionais sobre o Tamanho Médio de Partícula 63

6.2.1. Efeito da Temperatura sobre o Tamanho Médio de Cristalito 63

6.2.2. Efeito do Tempo Espacial sobre o Tamanho Médio de Cristalitos 65

6.3. Caracterização do pó sintetizado por Microscopia Eletrônica de Varredura (MEV) 66

6.4. Caracterização do pó sintetizado por Microscopia Eletrônica de
Transmissão (MET)

$\begin{array}{ll}7 . \text { CONCLUSÕES } & 79\end{array}$

8. RECOMENDAÇÕES PARA TRABALHOS FUTUROS 81

9 REFERÊNCIAS BIBLIOGRÁFICAS

10 APÊNDICES $\quad 87$

10.1 Apêndice I - Calibração do rotâmetro da Amônia 87

10.2 Apêndice II - Calibração do rotâmetro do primeiro cilindro de Argônio 88

10.3 Apêndice III - Calibração do rotâmetro do segundo cilindro de Argônio

89 


\section{Lista de figuras}

Figura 1 - Pó resultante da reação de $\mathrm{TiCl}_{4} \operatorname{com~} \mathrm{NH}_{3}$. .23

Figura 2 - Gráfico comparativo da pressão de vapor versus temperatura do $\mathrm{TiCl}_{4}$

e $\mathrm{AlCl}_{3}$.

Figura 3 - Composição de equilíbrio versus temperatura para o sistema reacional, mostrando a formação de TiN (sem supressão da decomposição de $\mathrm{NH}_{3}$ ).

Figura 4 - Composição de equilíbrio versus temperatura para o sistema reacional com a supressão da decomposição da amônia.

Figura 5 - Composição de equilíbrio versus temperatura para o sistema reacional Ti-O-N-H, para a formação de $\mathrm{TiO}_{2}$.

Figura 6 - Composição de equilíbrio versus temperatura para o sistema reacional $\mathrm{N}-\mathrm{H}$, mostrando a decomposição da amônia.

Figura 7 - Composição de equilíbrio versus temperatura para o sistema reacional $\mathrm{N}-\mathrm{H}-\mathrm{Cl}$, mostrando a decomposição do $\mathrm{NH}_{4} \mathrm{Cl}$. Comportamento do na faixa de temperatura entre 0 e $1000^{\circ} \mathrm{C}$

Figura 8 - Foto do aparato experimental que conduziu toda a experiência.......49

Figura 9 - Sistema de vaporização do tetracloreto de titânio 50

Figura 10 - Manta aquecedora - Sistema de vaporização do tetracloreto de titânio. 51

Figura 11 - Reator utilizado para a síntese de TiN..................................... 51

Figura 12 - Filtro para o recolhimento de ó. .52

Figura 13 - Coletor de pó mais solução de $\mathrm{NaHCO}_{3}$, para o tratamento de $\mathrm{HCl}$ .52

Figura 14 - Desenho esquemático do equipamento utilizado para a síntese de

TiN a partir da fase vapor.

Figura 15 - Gráfico Temperatura versus comprimento do forno $(\mathrm{cm})$ para o perfil

térmico na faixa entre 623 e $1173 \mathrm{~K}$. 55

Figura 16 - Difratograma de raios-X do pó depositado no papel de filtro a $800^{\circ} \mathrm{C}$

Figura 17 - Difratograma de raios-X de pó depositado na parede do tubo a $800^{\circ} \mathrm{C}$ 
Figura 18 - Difratograma de raios-X de pó depositado no papel de filtro a

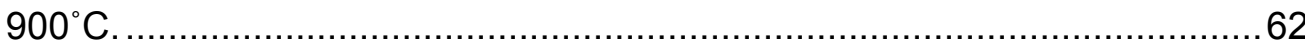

Figura 19 - Efeito da temperatura sobre o tamanho médio de cristalito. .............64

Figura 20 - Efeito do Tempo Espacial sobre o tamanho de cristalitos................66

Figura 21 - Micrografia (MEV) de aglomerados de partículas. .........................67

Figura 22 - Micrografia 01 de Ti-N-O para análise de EDS. ...........................67

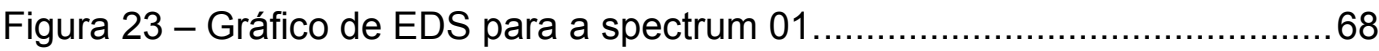

Figura 24 - Gráfico de EDS para o spectrum 02 ...........................................69

Figura 25 - (a) padrão de difração. (b) imagem em campo claro. (c) imagem em campo escuro centrado de uma amostra de TiN sintetizada a $900^{\circ} \mathrm{C}$..........70

Figura 26 - Distribuição do tamanho médio de partículas do TiN. O tamanho médio de partículas é de $13 \mathrm{~nm}\left({ }^{+} .5 \mathrm{~nm}\right)$ com um nível de confiança de

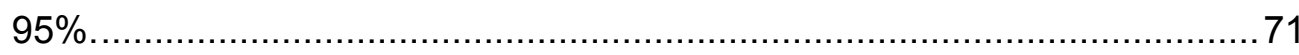

Figura 27 - Imagem em campo claro, que se observam planos atômicos. ........72

Figura 28 - Imagem em multi-feixe. ............................................................. 72

Figura 29 - (a) padrão de difração. (b) imagem em campo claro. (c) imagem em

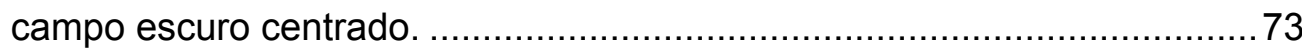

Figura 30 - Imagem de alta resolução - planos atômicos em algumas

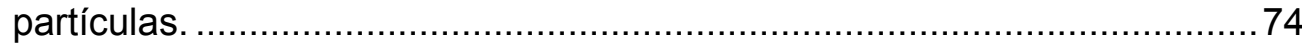

Figura 31 - Imagem de alta resolução mais bem definida - plano atômico.........74 Figura 32 - (a) padrão de difração. (b) imagem em campo claro. (c) imagem em campo escuro centrado de uma amostra sintetizada a $900^{\circ} \mathrm{C}$. .75

Figura 33 - (a) padrão de difração. (b) imagem em campo claro. (c) imagem em campo escuro centrado de uma amostra sintetizada a $900^{\circ} \mathrm{C}$. 76

Figura 34 - (a) Padrão de difração. (b) Imagem em campo claro. (c) Imagem em campo escuro centrado. (d) Imagem multi-feixe.....................................77

Figura 35 - Gráfico de EDS.obtido pelo MET. .......................................... 78

Figura 36 - Gráfico da calibração do fluxo de amônia na entrada do reator do sistema dinâmico na temperatura ambiente $\left(25^{\circ} \mathrm{C}\right)$..................................8 87

Figura 37 - Gráfico da calibração do fluxo do argônio na entrada do reator do sistema dinâmico na temperatura ambiente $\left(25^{\circ} \mathrm{C}\right)$................................... 88

Figura 38 - Gráfico da calibração do fluxo do argônio na entrada do reator do sistema dinâmico na temperatura ambiente $\left(25^{\circ} \mathrm{C}\right)$. .89 


\section{Lista de tabelas}

Tabela 1 - Propriedades do titânio (Lutjering \& Williams, 2002).

Tabela 2 - Propriedades comparativas do Titânio com outros materiais metálicos

(Lutjering \& Williams, 2002). 19

Tabela 3 - Reserva Mundial de produção e de reservas de $\mathrm{TiO}_{2}-2007$.

Tabela 4 - Produção de $\mathrm{TiO}_{2}$ em âmbito nacional - 2006.

Tabela 5 - Propriedades e características do TiN. 23

Tabela 6 - Propriedades do dióxido de titânio. 25

Tabela $7-\Delta G^{\circ}$ versus Temperatura. 38

Tabela 8 - Propriedades do $\mathrm{TiCl}_{4}$. 54

Tabela 9 - Resultados do estudo do efeito da variação da temperatura sobre o tamanho médio de cristalito $(\mathrm{nm})$. 63

Tabela 10 - Condições Reacionais para o Estudo da Variação do Tempo Espacial sobre o Tamanho de Cristalitos. 65

Tabela 11 - Resultados do Estudo do efeito da Variação do Tempo Espacial sobre o tamanho de cristalitos. 65

Tabela 12 - Tabela referente aos pontos de altura do rotâmetro versus vazão média (L/min).

Tabela 13 - Tabela referente aos pontos de altura do rotâmetro versus vazão média (L/min) para o primeiro cilindro de Ar. 88

Tabela 14 - Tabela referente aos pontos de altura do rotâmetro versus vazão média (L/min) para o segundo cilindro de Ar. 